\title{
Characterization of the Autographa californica Nucleopolyhedrovirus Ubiquitin Gene Promoter
}

\author{
$\mathrm{Xu}$ Ai Lin ${ }^{\mathrm{a}}$, Yin Chen ${ }^{\mathrm{a}}$, Wei Hua $\mathrm{Xu}^{\mathrm{b}}$, Yong Zhu Yia , and Zhi Fang Zhanga,* \\ a Biotechnology Research Institute, National Key Facility for Crop Gene Resources and \\ Genetic Improvement, Chinese Academy of Agricultural Sciences, Beijing, 100081, China. \\ Fax: +86-10-68975136. E-mail: zhifangzhang@yahoo.com \\ b State Key Laboratory for Biocontrol and Institute of Entomology, School of Life Sciences, \\ Zhongshan University, Guangzhou, 510275, China
}

* Author for correspondence and reprint requests

Z. Naturforsch. 63c, 277-283 (2008); received July 23/September 20, 2007

\begin{abstract}
Autographa californica multicapsid nucleopolyhedrovirus (AcMNPV) encodes an ubiquitin protein, which may be involved in virus infection. Functional analysis of the AcMNPV ubiquitin promoter was performed by progressive deletion of sequence or mutation of putative cis-activating motifs in the promoter region. In the presence of viral factors, a transient expression assay demonstrated that the active regions responsive to promoter transcription are mainly located within the range of -595 to -382 bp upstream of ATG. A 196-bp fragment ( -383 to $-187 \mathrm{bp}$ ), consisting of the distal TAAG, CAAT motif and TATA box, could also drive the expression of a reporter gene. Site-directed mutagenesis analyses indicated that mutations of TATA boxes and TAAG motifs reduce the promoter activity remarkably, while CAAT mutations enhance the promoter activity by about 3- or 4-fold as compared to the native promoter. All the results suggested that two continuous promoter regions are involved in the transcription of the ubiquitin gene and the cis-activating motifs corresponding to viral factors are mainly present within the $5^{\prime}$ region of the promoter. In addition, CAAT motifs in the promoter region function as negative regulator(s) binding sites.
\end{abstract}

Key words: Baculovirus, Ubiquitin Promoter, Transient Expression

\section{Introduction}

Baculoviruses are double-stranded, super-coiled DNA viruses. In cells infected with a baculovirus, viral genes are expressed in a temporarily controlled and sequentially ordered fashion. These genes have been classified as early, late or very late, based on their requirements for protein synthesis or viral DNA replication. Early gene promoter elements are similar to those from eukaryotic organisms and transcribed by the host RNA polymerase II (Hoopes and Rohrmann, 1991). Both late and very late genes are transcribed by an RNA polymerase complex mainly encoded by virus (Fuchs et al., 1983; Huh and Weaver, 1990), and initiated within or near the consensus sequence $(\mathrm{A} / \mathrm{G} / \mathrm{T})$ TAAG, which was referred to as a TAAG motif, conserved in distantly related baculoviruses and appeared to be a feature common to the baculoviridae family (Rohrmann, 1986).

Ubiquitin, a small protein, expresses in a wide variety of tissues and organisms from different species, with similar structure, function and immunological character (Hershko and Ciechanover,
1998). Some eukaryotic viruses, e.g. baculovirus, herpes virus and adenovirus, encode ubiquitin or ubiquitin-like proteins. They are not essential to virus replication but responsible for the virus infection, regulation of the immunity system of host cell and others (Ghosh et al., 2003). Autographa californica multicapsid nucleopolyhedrovirus (AcMNPV) encodes ubiquitin, which is transcribed during the late phase of virus infection, and there are two transcriptional start sites potentially encoding ubiquitin. The expressed protein is attached to membranes of virus particles and participates in the budding or production of virion (Guarino, 1990; Guarino et al., 1995; Reilly and Guarino, 1996). Thus, the investigation of viral ubiquitin will improve the recognition of viral evolution, infection and the interaction of virus and host.

In the present study we investigated the promoter activity upstream the ubiquitin gene in AcMNPV. A series of plasmids, containing fragments of different lengths upstream the AcMNPV ubiquitin ORF, was constructed and sequenced, respectively. Furthermore, a number of conserved 
motifs were site-directed mutated to investigate the transcriptional regulation of the ubiquitin promoter by these motifs using the luciferase gene as a reporter in insect cells through transient expression assays.

\section{Materials and Methods}

\section{Materials}

The Spodoptera frugiperda cell line $S f-21$ and pGEM-3Z vector were maintained in our laboratory. Plasmid pUL220, containing an entire luciferase gene (Lei et al., 1993), and wild-type AcMNPV were provided by Professor Xiangfu Wu (Shanghai Institute of Biochemistry, Chinese Academy of Sciences, Shanghai, China). TC-100 medium, fetal bovine serum (FBS) and lipofectin were purchased from Invitrogen (Carlsbad, CA, USA). Restriction endonucleases, high fidelity $p f u$ DNA polymerase, T4 DNA ligase, DNA purification kit and $\mathrm{pRL}-\mathrm{CMV}$ plasmid were obtained from Promega Corporation (Madison, WI, USA), unless otherwise stated. All chemicals were of analytical grade.

\section{Virus and cell culture}

The $S f-21$ cells were propagated with TC-100 medium containing 10\% FBS and penicillin G (80 $\mathrm{U} / \mathrm{ml})$-streptomycin $(50 \mu \mathrm{g} / \mathrm{ml})$ at $27^{\circ} \mathrm{C}$. AcMNPV virus was propagated in $S f$-21 cells, maintained at $27^{\circ} \mathrm{C}$ in TC-100 insect medium supplemented with $10 \%(\mathrm{v} / \mathrm{v})$ FBS. The details for cell culture, viral propagation, viral titer, and viral maintenance were according to Summers and Smith (1987).

\section{Construction of plasmids}

The reporter plasmids were generated to investigate the transient transcriptional activity of the baculovirus AcMNPV ubiquitin promoter. Primers for PCR amplification were designed based on the AcMNPV genomic sequence (GenBank accession number: NC001623). All the recombinant plasmids were constructed by inserting the promoter regions into the BamHI and EcoRI sites of pGEM-3Z plasmid.

Five clones were constructed to study the effects of deletions on the ubiquitin promoter region. Deletions, progressive removal nucleotides upstream of the ATG of ubiquitin, were obtained by the PCR method (primers are shown as in Table I). In the ubiquitin reverse primer, the start codon ATG was mutated to ATT for the construction of nonfused reporter plasmids. The obtained promoter regions had lengths of 595, 382, 124 and 26 base pairs (bp), respectively. All of the promoter regions included no less than one putative late promoter motif, TAAG, and TATA box situated from -19 to $-15 \mathrm{bp}$ upstream of the start codon ATG. Another fragment located -187 to $-383 \mathrm{bp}$ upstream of the ATG, containing a set of distal TAAG, CAAT motifs and TATA box, was also cloned by PCR.

To identify important regulatory motifs in the promoter region, mutations were introduced into the conserved elements region by PCR (primers

Table I. Primers used in the promoter deletion analysis.

\begin{tabular}{|c|c|c|}
\hline Name & Sequence (from $5^{\prime}$ to $3^{\prime}$ ) & Plasmid name \\
\hline Pubi F1 & TGGAATTCGATCGGATAGCGA & pAc595ubi \\
\hline Pubi F2 & CGGAATTCGTGTAACAAAGTCG & pAc382ubi \\
\hline Pubi F3 & TGGAATTCAATTGCCGTTGAAGGGAAAT & pAc124ubi \\
\hline Pubi R & TTTGGATCC TTACACTATTACTTATAAATGACAACGG & \\
\hline Pubi-2 ${ }^{\text {nd }} F$ & CGGAATTCGTGTAACAAAGTCGACAGCG & \multirow{2}{*}{ pAc196ubi } \\
\hline Pubi-2 ${ }^{\text {nd }} \mathrm{R}$ & GATGGATCCAAACGACTTAATATAACACC & \\
\hline Pcore F & 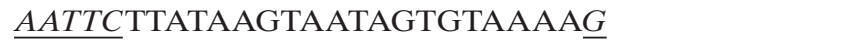 & \multirow{2}{*}{ pAc26ubi } \\
\hline Pcore R & $\overline{G A T C C}$ TTTTACACTATTACТTAТАA $\underline{G}$ & \\
\hline
\end{tabular}

Five clones were constructed to study the effect of deletions on the ubiquitin promoter transcription of AcMNPV using primers Pubi F1, Pubi F2, Pubi F3 and Pubi R to amplify the promoter fragments with the length of 595, 382 and $124 \mathrm{bp}$. Primers Pubi-2 ${ }^{\text {nd }} \mathrm{F}$ and Pubi- ${ }^{\text {nd }} \mathrm{R}$ were used to amplify a $196 \mathrm{bp}$ fragment containing the distal TAAG, CAAT motif and TATA box. Pcore F and Pcore R were used to synthesize a 26 bp promoter containing the proximal TAAG motif and TATA box. The EcoRI site in the forward primer and BamHI site in the reverse primer are in italics and underlined. 
Table II. Primers used in the promoter mutation analysis.

\begin{tabular}{clc}
\hline Name & Sequence (from 5' to $3^{\prime}$ ) & Plasmid name \\
\hline 17 M R & TTTGGATCCTTACACTATTACGGATAAATGACAACGG & p17Mubi \\
$195 \mathrm{M} \mathrm{F}$ & GTGGCCCGGTGTTATCCGTCGTTTGAAAGC & p195Mubi \\
$195 \mathrm{M} \mathrm{R}$ & GCTTTCAAACGACGGATAACACCGGGCCAC & p19Mubi \\
$19 \mathrm{M} \mathrm{R}$ & TTTGGATCCTTACACTATTACTTACGAATGACAACGG & p269Mubi \\
$269 \mathrm{M} \mathrm{F}$ & CGTCAGCCTCCGTAATCTCGG & \\
$269 \mathrm{M} \mathrm{R}$ & CCGAGATTACGGAGGCTGACG & p71Mubi \\
$71 \mathrm{M} \mathrm{F}$ & CGCCTGTTGCACGGTTCCACCAGCGGTG & \\
$71 \mathrm{M} \mathrm{R}$ & CACCGCTGGTGGAACCGTGCAACAGGCG & p359Mubi \\
$359 \mathrm{M} \mathrm{F}$ & GACAGCGAAACGGTTTATCGTTTTC & \\
$359 \mathrm{M} \mathrm{R}$ & GAAAACGATAAACCGTTTCGCTGTC &
\end{tabular}

Several mutations were conducted in the putative motifs by the PCR method. TATA boxes, CAAT and TAAG motifs were mutated to CGTA, CGGT and TCCG, respectively. For mutation, the forward and reverse primers were reversely complemented. Pubi F1 and every mutated reverse primer were used to amplify the distal fragment. Every mutated forward primer and Pubi R primer were used to amplify the proximal fragment with Pfu DNA polymerase. After recovered from gel, the distal and proximal fragments were allowed to anneal, and then the primers Pubi F1 and Pubi R were employed to amplify the full promoter region with the desired mutation. The mutated nucleotides are in italics and underlined. All the mutated sequences were confirmed by sequencing.

used as shown in Table II). A mutated site was designed in the middle of each mutated primer and two primers, containing the same mutated site, were complementary reversed. Pubi F1 and the mutated reverse primer were used to amplify the fragment containing the mutated site in the $3^{\prime}$ end; the mutated forward primer and Pubi R was used to amplify the fragment containing the mutated site in the $5^{\prime}$ end. An annealed two-corresponding fragment, containing the same mutated site, was subsequently added to Pubi F1 and Pubi R to amplify the promoter fragment, which contained a site-directed mutation of an involved motif, such as TAAG, CAAT or TATA box. In this experiment, TATA boxes and TAAG motifs were mutated to CGTA and TCCG, respectively, and CAAT motifs were mutated to CGGT. All mutated sequences were confirmed by sequencing.

The entire coding region of the luciferase gene was separated from the plasmid pUL220 and subcloned into the BamHI site of every plasmid, under the control of the regenerated promoters in right orientation.

All the general approaches were performed as described previously (Sambrook et al., 1989).

\section{Transfection in insect cells and transient expression analysis}

The effect of deletions or mutations on the promoter region of the ubiquitin gene was investi- gated by a transient expression assay. For transfection, $S f-21$ cells were seeded in a $15 \mathrm{~cm}^{2}$ flask at a density of about $1 \cdot 10^{6}$ cells $/ \mathrm{ml}$, and cells were allowed to attach to the flask at $27^{\circ} \mathrm{C}$ overnight. TC-100 medium was then replaced by $1 \mathrm{ml}$ of serum-free medium and $50 \mu \mathrm{l}$ of transfection solution containing $5 \mu \mathrm{l}$ of lipofectin, $1 \mu \mathrm{g}$ of one of the reporter plasmid DNA described above, and $0.1 \mu \mathrm{g}$ of control plasmid, pRL-CMV (Promega). $3 \mathrm{~h}$ post transfection (h.p.t.), followed by infection of wild-type AcMNPV (MOI $=1.0)$ for $1 \mathrm{~h}$, the supernatant was replaced with $3 \mathrm{ml}$ of conditioned medium. Each treatment consisted of at least three separate transfections.

The transfected $S f$-21 cells were harvested at 48 h.p.t. by centrifugation, and cell extracts were prepared using a dual luciferase assay kit (Promega). The harvested cells were washed twice by resuspending them in phosphate buffered saline (PBS) and centrifugation at $6,000 \times g$ for $5 \mathrm{~min}$ at $4{ }^{\circ} \mathrm{C}$. After washing, the cells were lysed by passive lysis buffer and a single freeze-thawing cycle. Before the measurement, the lysate was centrifuged at $4{ }^{\circ} \mathrm{C}$ to remove cell debris.

Measurements of luciferase activity from three separate transfections were done in triplicate using a liquid scintillation spectrometer (Beckman, Fullerton, CA, USA). For normalization of the luciferase activity from each transfection, an internal control plasmid pRL-CMV was used, which contained the Renilla luciferase gene under the con- 
trol of the CMV promoter. The amount of protein in the lysate was measured as described by Bradford (1976).

\section{Results}

Cloning and sequence analysis of baculovirus ubiquitin promoter

Primers were designed according to the reported sequence of AcMNPV. In the reverse primer, the start codon ATG was mutated into ATT to construct non-fused reporter plasmids. Using genomic DNA of wild-type AcMNPV as template, a 595-bp fragment upstream of ubiquitin ORF was amplified by PCR. The products were recovered from agarose gel and inserted into the BamHI and EcoRI sites of pGEM-3Z.

The cloned fragment of the promoter region was sequenced and aligned with the homologous promoter sequence of AcMNPV C6 strain. The result showed that the cloned promoter region was identical to the corresponding region of the C6 strain. In the promoter region, there were two putative TATA boxes at $-19 \mathrm{bp}$ and $-269 \mathrm{bp}$, and two putative CAAT motifs at $-71 \mathrm{bp}$ and $-359 \mathrm{bp}$, respectively; the late transcription start site, TAAG, was located at $-17 \mathrm{bp}$ and $-195 \mathrm{bp}$, with respect to the translation start site ATG.

\section{Transcription activity of ubiquitin promoter}

$S f$-21 cells were transfected for $4 \mathrm{~h}$ with a mixture of lipofectin and reporter plasmid, pAc595ubi-luc, as well as normalization plasmid pRLCMV. The transfected cells were harvested 48 h.p.t. The luciferase activity was indicated as counts per min (cpm) in $15 \mathrm{~s}$ of $20 \mu \mathrm{g}$ protein extracted from $S f-21$ cells. The wild-type AcMNPV infected cells or cells transfected with pUL220 plasmid were employed as controls. The luciferase activity in uninfected cell extracts was about 16 cpm, equally to the extracts from wild-type AcMNPV infected cells or pUL220 transfected cells (background value). Contrarily, in the cell extracts infected by AcMNPV, the luciferase activity was $(27588 \pm 664.87) \mathrm{cpm}$. This result indicated that the luciferase gene is not expressed in noninfected cells. The transcription of the ubiquitin promoter was dependent on the virus infection. The ubiquitin gene may be a late viral gene and certain factor(s) encoded by the virus contributed to the activation of the ubiquitin promoter.

\section{Effect of deletions on the promoter activity}

A series of plasmids containing the luciferase gene under the control of the ubiquitin promoter was constructed, in which the ubiquitin promoter

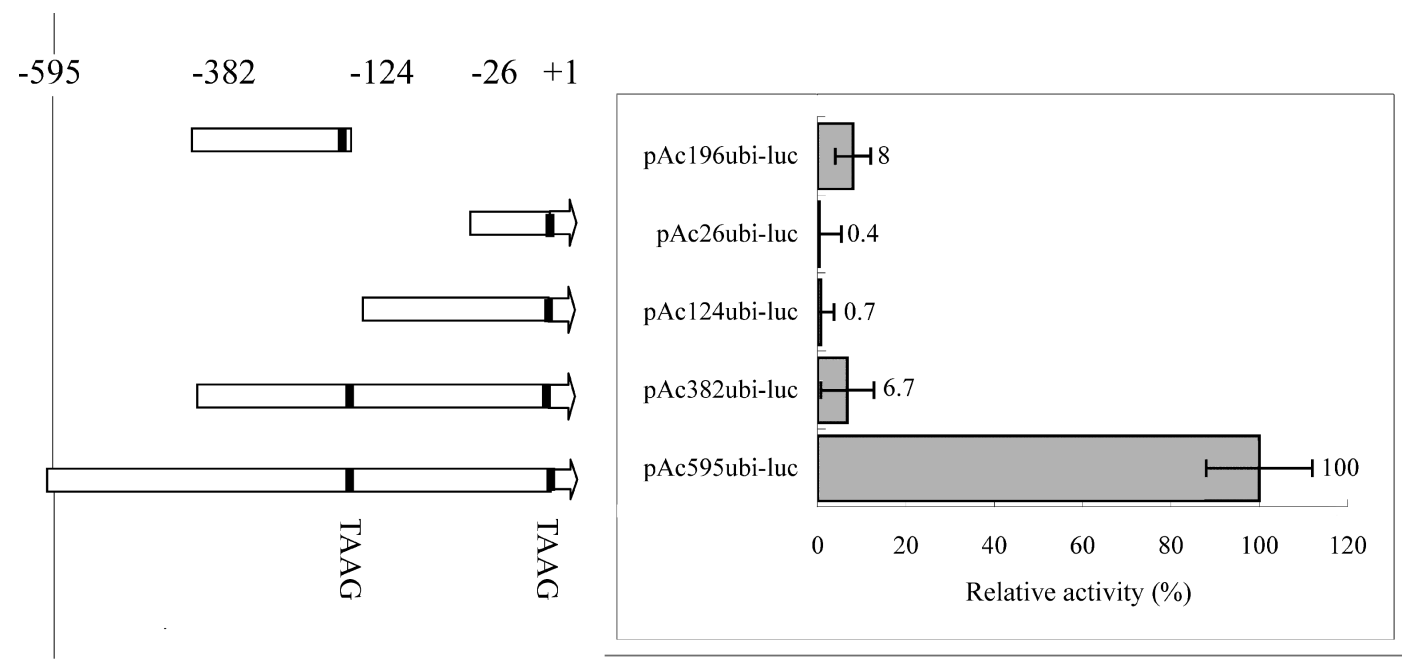

Fig. 1. Relative activity of luciferase controlled by the different length of the ubiquitin promoter. $S f$-21 cells were transfected with $1 \mu \mathrm{g}$ of each plasmid DNA, followed by an infection of wild-type AcMNPV, and incubated for $48 \mathrm{~h}$ at $27^{\circ} \mathrm{C}$. Transcription activity of each promoter is presented as percentage over the full-length promoter that is arbitrarily set as 100. The wild-type AcMNPV genomic DNA was used as template in PCR reactions to obtain promoter fragments with different lengths. Five plasmids were constructed by inserting the PCR products of appropriate length in pGEM-3Z. The relative activities of the corresponding plasmids are presented in the right half of the figure. The TAAG motif in the promoter region of the inserts is marked by a black box. 
was progressively deleted at the $5^{\prime}$ region by PCR. The effect of these deletions on the ubiquitin promoter activity was determined by transfecting each plasmid into $S f$-21 cells. In each treatment, pRL-CMV was employed as internal control plasmid to normalize the luciferase activity of the cell extracts obtained from the transfected cells. The promoter activity would be indicated by the expression of the luciferase gene from each deleted promoter. In the presence of virus factors, the transcriptional activity of the 595-bp promoter was highest in all generated promoters. The activity decreased remarkably and remained at about $6.7 \%$ activity, when the deletion occurred at 595 to 382 bp upstream ATG. However, when the promoter was only 124 bp or 26 bp in length, its activity was almost abolished. But the region, which included another set of the putative TATA box, CAAT and TAAG motifs, from -383 to $-187 \mathrm{bp}$ with respect to the translation start codon, could also drive the expression of luciferase in a transient expression assay. Fig. 1 summarizes the results obtained from the deletion analysis.

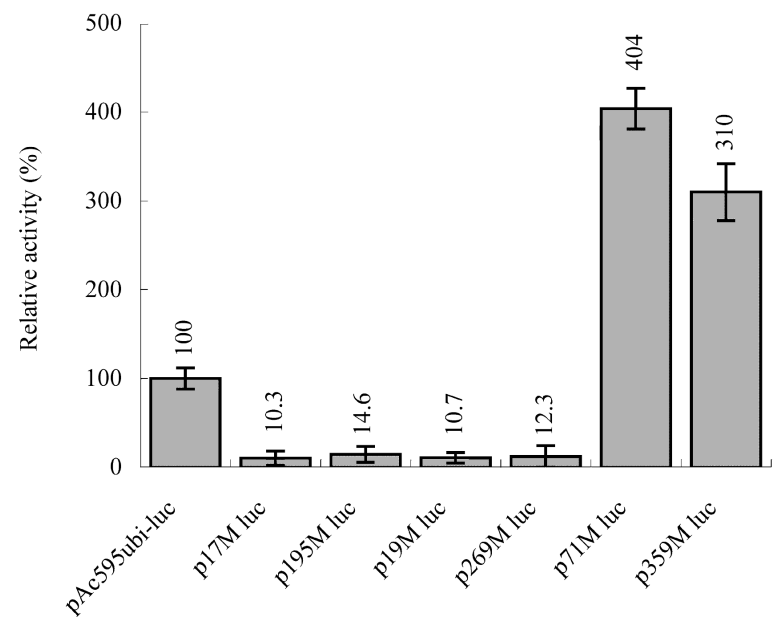

Fig. 2. Relative activity of luciferase controlled by the mutated ubiquitin promoter. $S f$-21 cells were transfected with $1 \mu \mathrm{g}$ of each plasmid DNA, followed by an infection of wild-type AcMNPV, and incubated for $48 \mathrm{~h}$ at $27^{\circ} \mathrm{C}$. Transcription activity of each promoter is presented as percentage over the full-length promoter that is arbitrarily set as 100 . The putative function sites in the promoter region were separately tested by mutating each site in the promoter region. The sequence of the pAc595ubiluc plasmid was used as PCR template to obtain the promoter fragment containing mutated TAAG, TATA or CAAT, respectively.

\section{Effect of site-directed mutagenesis on the promoter activity}

The modifications also include mutations of the TATA box, TAAG, CAAT motifs within the ubiquitin promoter region. In this experiment, the TAAG motifs, TATA boxes and CAAT motifs were mutated to TCCG, CGTA and CGGT, respectively. The results revealed that mutations of both TATA box and TAAG motifs could reduce the promoter activity remarkably to about $10 \%$ residual activity, while the CAAT mutation could up-regulate the promoter activity by about 3- or 4 -fold compared to the native promoter. The results are presented in Fig. 2.

\section{Discussion}

In order to study the characteristics of the ubiquitin promoter, $S f$-21 cells were co-transfected with reporter plasmids followed by infection with wild-type AcMNPV. The transient expression assay revealed that deletion between -595 to -382 bp relative to ATG significantly reduced the promoter activity compared to the full-length promoter (595 bp), but there was no remarkable reduction of the promoter activity by further deletions. In addition, we cloned another 196-bp fragment situated from -383 to $-187 \mathrm{bp}$ in the promoter region, which paralleled the second deletion and contained a set of the distal TATA box, TAAG and CAAT motifs. The luciferase activity was comparable with the second deletion (382 bp long promoter), which contained both the distal and proximal motifs. All these results suggested that cis-activating elements responsive to viral factors are mainly located within the range from 595 to 382 bp upstream ATG, and another fragment that contained distal TAAG and CAAT motifs and the TATA box also could function as a promoter. This result may support another evidence that ubiquitin has two transcriptional initiation sites (Guarino, 1990).

The transcription initiation site, TAAG, of baculovirus late and very late genes plays one or more roles in late and very late gene expression. It may serve to stabilize the RNAs or facilitate translation. Another role of the TAAG site is its function in RNA initiation by binding a transcriptional activator or RNA polymerase (Funk et al., 1998). A previous work demonstrated that TAAG and its flanking sequence have significant influence on AcMNPV vp39 promoter activity (Morris and Mil- 
ler, 1994). In the 5' region of the AcMNPV ubiquitin promoter, two TAAG motifs are located at 17 and 195 bp upstream ATG, respectively. In this experiment, both the distal and proximal mutations of putative TAAG motifs led to a remarkable reduction of promoter activity. Perhaps the mutation of TAAG changed the recognizable site of RNA polymerase. The TAAG at $-17 \mathrm{bp}$ was overlapping with the TATA box; they formed the sequence TATAAG, so the mutation of TAAG might affect the construction of the TATA box, and led to an incorrect reorganization of RNA polymerase.

Gene expression and regulation are mediated by a DNA sequence upstream of the coding sequences, the so-called promoter fragment. This DNA sequence provides gene expression regulation at the stage of transcription initiation, which contains numerous short functional elements, the transcription factor binding sites, which function as recruiting transcription factors, as regulators, and as an RNA polymerase. Generally, a promoter contains two primary motifs, TATA box and CAAT motif. The TATA box assists in directing RNA polymerase II to the transcription initiation site. It locates at a relatively fixed position upstream of the transcription initiation site. Mutation of the TATA box would lead to the incorrect initiation of mRNA. The result of this experiment showed that the TATA box mutation greatly reduces the expression of the reporter gene, which indicates that the TATA box plays an essential role in the regulation of the ubiquitin promoter.

In addition, the CAAT motif is another highly conserved transcriptional motif present in the promoter region, which is one of the binding sites of RNA polymerase. Its function in the promoter is

Bradford M. M. (1976), A rapid and sensitive method for the quantitation of microgram quantities of protein utilizing the principle of protein-dye binding. Anal. Biochem. 72, 248-254.

Fuchs L. Y., Woods M. S., and Weaver R. F. (1983), Viral transcription during Autographa californica nuclear polyhedrosis virus infection: a novel RNA polymerase induced in infected Spodoptera frugiperda cells. J. Virol. 38, 641-646.

Funk C. J., Harwood S. H., and Rohrmann G. F. (1998), Differential stability of baculovirus late transcription complexes during initiation and elongation. Virology 241, $131-140$. still unknown; previous research evidenced that it controls the frequency of transcriptional initiation. In this study, the mutation of CAAT elevated the promoter activity by about 3 or 4 times relative to the native promoter. It is assumed that the CAAT motif negatively regulates the ubiquitin promoter activity. We presumed that certain negative regulating protein(s) combined with the CAAT motif to down-regulate the transcription, while the CAAT mutation could avoid the binding of negative regulating protein(s) and as a result enhanced the activity of the transcription of the promoter. Previous genetic analysis of the CAAT motif showed that a single point mutation in the $5^{\prime} \mathrm{A}$ residue has significant phenotypic effects when coupled with otherwise transcriptionally silent mutations in the upstream promoter element or TATA box (Maity et al., 1988). Reach and his coworkers also confirmed the previous observation that there was functional redundancy between the two upstream activating elements (Reach et al., 1991).

In this experiment, we found that the cis-activating elements of the ubiquitin promoter has differential functions. The transient expression assays indicated that a mutation of both TATA boxes and TAAG motifs reduces the ubiquitin activity significantly, while the mutation of the CAAT motif could increase the promoter activity. All these motifs function simultaneously to ensure the precise regulation of the ubiquitin gene in the host cells.

\section{Acknowledgements}

This work was supported by financial grants from The National Natural Science Foundation of China (No. 30670082) and National Basic Research Program of China (973 Program, No. 2005CB121006).

Ghosh M., Huang K., and Berberich S. J. (2003), Overexpression of Mdm2 and MdmX fusion proteins alters p53 mediated transactivation, ubiquitination, and degradation. Biochemistry 42, 2291-2299.

Guarino L. A. (1990), Identification of a viral gene encoding a ubiquitin-like protein. Proc. Natl. Acad. Sci. USA 87, 409-413.

Guarino L. A., Smith G., and Dong W. (1995), Ubiquitin is attached to membranes of baculovirus particles by a novel type of phospholipid anchor. Cell 80, 301-309.

Hershko A. and Ciechanover A. (1998), The ubiquitin system. Annu. Rev. Biochem. 67, 425-479. 
Hoopes R. and Rohrmann G. (1991), In vitro transcription of baculovirus immediate early genes: accurate mRNA initiation by nuclear extracts from both insect and human cells. Proc. Natl. Acad. Sci. USA 88, $4513-4517$.

Huh N. M. and Weaver R. F. (1990), Identifying the RNA polymerases that synthesize specific transcripts of the Autographa californica nuclear polyhedrosis virus. J. Gen. Virol. 71, 195-201.

Lei X. D., Mi Y. D., Wu X. F., Li Z. P., and Yuan Z. Y. (1993), Expression of luciferase gene in insect cells. Acta Biochim. Biophys. Sin. 25, 65-69.

Maity S. N., Golumbek P. T., Karsenty G., and de Crombrugghe B. (1988), Selective activation of transcription by a novel CCAAT binding factor. Science 241, $582-585$.

Morris T. D. and Miller L. K. (1994), Mutational analysis of a baculovirus major late promoter. Gene $\mathbf{1 4 0}$, $147-153$.
Reach M. L., Xu X. C., and Young S. H. (1991), Transcription from the adenovirus major late promoter uses redundant activating elements. EMBO J. 10, 3439-3446.

Reilly L. M. and Guarino L. A. (1996), The viral ubiquitin gene of Autographa californica nuclear polyhedrosis virus is not essential for viral replication. Virology 218, 243-247.

Rohrmann G. F. (1986), Polyhedrin structure. J. Gen. Virol. 67, 1499-1513.

Sambrook J., Fritsch E. F., and Maniatis T. (1989), Molecular Cloning: A Laboratory Manual, 2nd ed. Cold Spring Harbor Laboratory Press, Cold Spring Harbor, NY.

Summers M. D. and Smith G. E. (1987), A Manual of Methods for Baculovirus Vectors and Insect Cell Culture Procedures. Texas Agricultural Experiment Station Bulletin, No. 1555, College Station, TX, USA. 